\title{
Estimation of particle size distribution from bulk scattering spectra: validation on monomodal suspensions
}

\author{
Annelies Postelmans, ${ }^{\dagger}$ Ben Aernouts, ${ }^{\dagger}, \ddagger$ and Wouter Saeys ${ }^{*, \dagger}$ \\ $\dagger$ Department of Biosystems, MeBioS, KU Leuven, Kasteelpark Arenberg 30, 3001 Leuven, \\ Belgium \\ $\ddagger$ Department of Microbial and Molecular systems, Cluster for Bioengineering Technology, \\ KU Leuven Campus Geel, Kleinhoefstraat 4, 2440 Geel, Belgium \\ E-mail: wouter.saeys@kuleuven.be
}

\section{Abstract}

A particle size distribution (PSD) estimation method based on light scattering properties was validated on experimental Vis/NIR scattering spectra of polystyrene suspensions, with a nominal particle size ranging from 0.1 to $12 \mu \mathrm{m}$ in diameter. Based on $\mu_{s}$ and $g$ spectra extracted from double integrating sphere measurements, good PSD estimates were obtained for particles $\geq 1 \mu \mathrm{m}$. The particle volume fraction estimates in case of $\mu_{s}$ were close to the target concentrations, although influenced by small baseline fluctuations on the spectra. For submicron particles, on the other hand, the non-oscillating $\mu_{s}$ spectra lack discriminating power, resulting in erroneous PSD estimates. The reduced scattering coefficient spectra $\left(\mu_{s}^{\prime}\right)$ were found less useful for particle size estimation as they lack a characteristic shape, causing an over- or underestimation of the distribution width. In summary, the estimation routine proved to deliver PSD estimates in line with the reference measurements for micron-sized or larger particles based on their $\mu_{s}$ and $g$ scattering spectra. Additional validation on more polydisperse samples forms the next step before going to bimodal PSD estimates.

Particle size measurements are an important tool for characterization and quality control of turbid media. Currently, a variety of bench-top sizing instruments exist, which exploit the interaction of particles with light (e.g. dynamic light scattering (DLS), laser diffraction (LD) or focused beam reflectance measurements), the differences in electrical conductivity such as electrical sensing zone (ESZ), or image analysis based methods like (nano)particle tracking analysis. ${ }^{1-3}$ Each of these techniques has an optimal particle size working range and certain requirements for the samples which can be measured. Depending on the instrument software, prior information on e.g. monomodality or expected width of the PSD can be incorporated. With respect to the particle size range, DLS is more suitable for submicron particles, while LD performs better for particles $>1 \mu \mathrm{m}$, although a correct choice has to be made for the optical model (Mie theory or Fraunhofer diffraction). ${ }^{2}$

In general, these techniques analyse rather small, (strongly) diluted samples. While the small volume might not be representative for the entire sample, the strong dilution can disrupt clusters or aggregates that were present in the original sample. ${ }^{4}$ Due to this, the measured PSDs may vary strongly depending on the used sizing instrument and settings.

A large number of particle sizing techniques are based on the interaction of light with particles. This has some similarities with the 
Vis/NIR spectroscopy techniques which are widely used for process monitoring and control. ${ }^{5}$ Such spectroscopic measurements are influenced by the chemical content (related to light absorption) and microstructure (related to light scattering). ${ }^{6}$ However, classical chemometrics focuses mainly on absorption characteristics, while scattering effects are often regarded as interfering and therefore removed by (semi-empirical) preprocessing. ${ }^{7,8}$ Some researchers have trained multivariate calibration models like partial least squares regression for estimating the mean particle size or a related parameter directly from the acquired spectra. ${ }^{9}$ However, they were not able to predict a complete PSD and their databased models can only be used on samples very similar to the ones used for training. ${ }^{10-13}$

Information on light scattering and absorption can be derived from diffuse reflectance and transmittance spectra of turbid samples. $^{6,8,14,15}$ These bulk optical properties (BOP) include the wavelength dependent absorption coefficient $\mu_{a}$, scattering coefficient $\mu_{s}$ and anisotropy factor $g$. The two latter can be combined into the reduced scattering coefficient $\mu_{s}^{\prime}$. To determine BOP experimentally, multiple spectroscopic measurements, such as in spatially resolved spectroscopy or integrating sphere measurements, are combined with light propagation models to link the obtained reflection and/or transmission spectra to optical properties. ${ }^{16}$ Steponavicius \& Thennadil used this approach to obtain pure absorption spectra for improved quantitative analysis of different components in a turbid mixture. ${ }^{14,17}$ On the other hand, the bulk scattering properties contain direct information on the microphysical properties such as particle size, concentration, shape and material.

Recently, we presented a method to estimate monomodal PSDs from $\mu_{s}, \mu_{s}^{\prime}$ and $g$ spectra and evaluated its sensitivity for different error sources through simulations. ${ }^{18}$ It was demonstrated that this routine can provide accurate estimates for both the PSD parameters and the volume fraction (VF) of particles, without the need for severe sample dilution. Although the added noise was selected to mimic differ- ent types of measurement noise, the estimation routine was not yet confronted with experimental scattering spectra. Such spectra typically contain an unknown combination of errors resulting from sample preparation, set-up specific noise and uncertainties on input parameters such as the sample's refractive index, which makes it difficult to capture the full complexity in simulations.

Therefore, the aim of this study was to validate this method for estimating the monomodal PSDs and particle concentrations of polystyrene suspensions (often used as model system) from the bulk scattering properties obtained by double integrating sphere measurements. The PSD estimates were compared to PSDs obtained with commercially available bench-top particle sizing instruments.

\section{Materials and methods}

\section{Samples and reference PSDs}

Suspensions of monomodal polystyrene particles in water (micromer ${ }^{\circledR}$ ) with nominal diameters of $0.1,0.5,1,3,6,10$ and $12 \mu \mathrm{m}$ were purchased from micromod Partikeltechnologie GmbH (Germany). The stock suspensions were placed in a rotor at $30 \mathrm{rpm}$ at room temperature three hours prior to the optical measurements until the end of the optical measurements. They were diluted to subsamples with nominal concentrations of $0.75,1$ and $1.25 \%$ $\mathrm{v} / \mathrm{v}$ right before loading them into the cuvettes, always in four independent replicates.

Volume based PSDs of the $0.1,0.5$ and $1 \mathrm{\mu m}$ particles were measured by the manufacturer with DLS on a Zetasizer Nano ZS 90 (Malvern instruments, UK). For the 3, 6, 10 and $12 \mu \mathrm{m}$ particles, they measured number based PSDs with ESZ (Multisizer 3, BeckmanCoulter, USA), which were afterwards converted to volume based PSDs. The PSDs of the samples were measured a second time by the authors with DLS for the $0.1-1 \mu \mathrm{m}$ particles with setting 'narrow mode' (Zetasizer Nano ZS, Malvern instruments, UK), and with LD for 0.5-12 $\mu \mathrm{m}$ particles (Mastersizer 3000, Malvern 
Table 1: List of abbreviations and symbols.

\begin{tabular}{|ll|ll|}
\hline \multicolumn{2}{|c|}{ Abbreviations } & \multicolumn{1}{c|}{ Symbols } \\
\hline BOP & bulk optical properties & $\mu \& \sigma$ & (log)normal \\
DLS & dynamic light scattering & & distribution parameters \\
ESZ & electrical sensing zone & $\mu_{s}$ & scattering coefficient \\
FWHM & full width at half maximum & $\mu_{s}^{\prime}$ & reduced scattering coefficient \\
LD & laser diffraction & $a \& b$ & Weibull distribution parameters \\
PSD & particle size distribution & $g$ & anisotropy factor \\
RMSE $(\mathrm{CV})$ & root mean squared error & $n$ & real refractive index \\
& (of cross-validation) & par $1 \&$ par 2 & generic distribution parameters \\
Vis/NIR & visible \& near infrared & $R^{2}$ & coefficient of determination \\
VF & volume fraction & & \\
\hline
\end{tabular}

instruments, UK). For PSD determination with LD, two different analysis models were used: 'narrow modes' (LD-Narrow) and 'verification latex' (LD-Latex). These analysis models were preferred over the 'general mode' since the particles were known to be monomodal with a narrow distribution based on the manufacturer's specifications. ${ }^{19}$ The PSDs were well within the specified range for the respective instruments. ${ }^{20-22}$ For comparison of PSD estimates with these references in the results section, only the manufacturer's PSD will be shown because of the similarity of simulated spectra and those estimated from double integrating sphere measurements, as discussed in the section Simulated \& estimated BOP. The reference PSDs are shown in interpolated (and smoothed in case of 3-12 $\mathrm{mm}$ ) form instead of histograms.

\section{Forward simulation of BOP}

BOP spectra, $\mu_{s}, \mu_{s}^{\prime}$ and $g$, of all reference PSDs were calculated based on Mie theory using the microscale tool developed by Aernouts et al. ${ }^{23}$ The spectra were simulated over the 0.5-1.85 $\mathrm{\mu m}$ wavelength range with a $10 \mathrm{~nm}$ step. The complex refractive index values of water reported by Segelstein were used for the medium. ${ }^{24} \mathrm{~A}$ fit of the Sellmeier dispersion equation to the refractive index values of polystyrene determined by Sultanova et al. ${ }^{25}$ was used as real particle refractive index. ${ }^{26}$ The imaginary part of the particle refractive index was assumed to be zero since Ma et al. mention a transparent region for polystyrene from
390-1310 nm, and Inagaki et al. even up to $2066 \mathrm{~nm} .{ }^{27,28}$ Furthermore, the effect of absorption is negligible if the imaginary part of the refractive index is (very) small compared to the real part. ${ }^{29}$

A particle concentration of $1 \% \mathrm{v} / \mathrm{v}$ was used for all simulations since the BOP could easily be converted to the level of $0.75 \%$ and $1.25 \% \mathrm{v} / \mathrm{v}$ through scaling. That is, due to the independent scattering assumption, $\mu_{s}$ and $\mu_{s}^{\prime}$ are linearly proportional to the particles concentration. The anisotropy factor $g$ is concentration independent.

\section{BOP from measurements}

The BOP of the polystyrene samples were estimated from total reflectance and total transmittance data obtained by means of a double integrating sphere setup and unscattered transmission measurements, using the setup described by Aernouts et al. ${ }^{30}$ A simplified schematic representation is given in Fig. 1. In further reference, they will be labelled 'estimated' BOP. The samples were loaded into borosilicate $\mathrm{cu}$ vettes and placed in a sample holder sandwiched between two integrating spheres. The wavelengths ranged from 0.5 to $1.85 \mu \mathrm{m}$ with a $10 \mathrm{~nm}$ step. For 10 and $12 \mu \mathrm{m}$ particles, some sedimentation in the cuvettes was noticed during the measurements. However, the effect was minimal as there was no sloped baseline visible in the $\mu_{s}$ spectra, which would have indicated a changing particle concentration during the measurement ( \pm 2 min.). This was verified 
by comparing the estimated BOP with simulated spectra, and additional stability measurements at $800 \mathrm{~nm}$ wavelength ( $\pm 5 \mathrm{~min}$.).

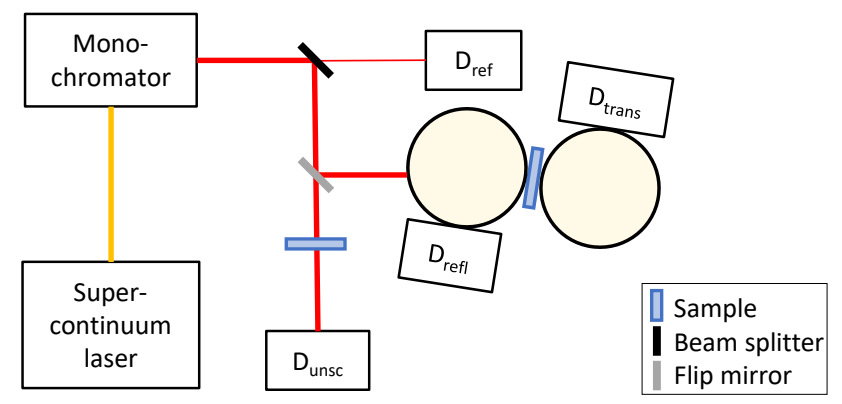

Figure 1: Simple scheme of the double integrating sphere setup of Aernouts et al. ${ }^{30} \mathrm{D}=$ detectors; ref $=$ reference, refl $=$ total reflectance, trans $=$ total transmittance; unsc $=$ unscattered transmission.

The BOP were calculated from the reflectance and transmittance spectra using the inverse adding doubling algorithm as implemented by Prahl. ${ }^{31}$ In addition to the integrating sphere characteristics, thickness of the cuvette walls and refractive index of the cuvette glass, the sample thickness and the wavelength dependent real refractive index of the sample $(n)$ was given as input (Eq. (1)).

$$
\begin{aligned}
n_{\text {sample }}=n_{\text {medium }}+ & \% v / v_{\text {particles }} \times \\
& \left(n_{\text {particles }}-n_{\text {medium }}\right)
\end{aligned}
$$

All spectra were preprocessed before PSD estimation. First, a baseline was added to the spectra for wavelengths $>1.05 \mu \mathrm{m}$, proportional to the small jump in signal at 1.05-1.06 $\mu \mathrm{m}$ due to the switch of detector type. ${ }^{30}$ No baseline was added to wavelengths $<1.05 \mu \mathrm{m}$, because this part had the most correct spectral level based on comparison with simulated spectra. After that, spectra were smoothed by a third order Savitzky-Golay filter with frame length 9 .

The high absorption at $1450 \mathrm{~nm}$ by the O$\mathrm{H}$ bonds in water resulted in very low total reflectance and transmittance signals. This complicated the estimation of the absorption and scattering coefficients at this wavelength. Therefore, $\mu_{s}$ spectra were used from 0.5-1.35 and 1.55-1.85 $\mathrm{\mu m}$ for PSD estimation. Additionally, only $\mu_{s}$ values $>0.15 \mathrm{~mm}^{-1}$ were considered as lower values were unreliable due to a low signal to noise ratio. In case of $g$ and $\mu_{s}^{\prime}$, higher wavelengths were also affected by noise and therefore these spectra were only used in the range $0.5-1.35 \mu \mathrm{m}$, and for $\mu_{s}^{\prime}>0.1 \mathrm{~mm}^{-1}$.

\section{Monomodal shape dependent PSD estimation}

Monomodal shape dependent PSD estimations were made based on $\mu_{s}, \mu_{s}^{\prime}$ and $g$ spectra. Three types of 2-parameter probability density functions (lognormal, normal and Weibull) were considered as reasonable approximations for the real PSD shape. For every sample, PSD estimates for these three distribution types were compared and the type with the lowest cost (Eq. (2)) was selected as final PSD estimate. The distribution parameters were estimated based on the shape of the scattering spectra, because spectral shape is mainly determined by particle size. Therefore, all spectra were normalized through division by the spectral mean over all wavelengths. Note that $\mu_{s}$ in Eq. (2) can be replaced by $g$ or $\mu_{s}^{\prime}$. The normalisation eliminated the VF dependency of $\mu_{s}$ and $\mu_{s}^{\prime}$ spectra. Moreover, it reduced the effect of baseline errors on $g$ spectra. $N_{\lambda}$ is the total number of wavelengths of the respective scattering spectrum. Par 1 and par 2 represent the distribution parameters $\mu$ and $\sigma$ of a (log)normal distribution, or the scale and shape parameters $a$ and $b$ of a Weibull distribution.

$\min \log _{10}\left[\sum_{i=1}^{N_{\lambda}}\left(\frac{\frac{\mu_{s, i}}{\operatorname{mean}\left(\mu_{s}\right)}-\frac{\widehat{\mu_{s, i}}(\text { par } 1, \text { par } 2)}{\operatorname{mean}\left(\widehat{\mu_{s}}(\text { par } 1, \text { par } 2)\right)}}{\frac{\mu_{s, i}}{\operatorname{mean}\left(\mu_{s}\right)}}\right)^{2}\right]$

To decrease the possibility of selecting a local minimum as final solution, a multistart approach was used. First, the cost function was evaluated on a 100x100 grid (linear spacing) spanning the complete distribution parameter space (Table 2). Regional minima in this grid were detected using 'imregionalmin' in Matlab, and served as initial points for the non-gradient based optimization ('patternsearch'). A selec- 
tion procedure was applied to the optimization end points to exclude solutions

- that did not converge,

- with a positive cost (corresponding to a sum of squared relative errors $>10$ ),

- that were positioned on a distribution parameter boundary,

- with a ratio of the mean input and estimated spectra smaller or larger than respectively the lower or upper VF boundary in case of $\mu_{s}$ and $\mu_{s}^{\prime}$ (to prevent subsequent $\mathrm{VF}$ estimates from reaching the VF boundaries),

- with a ratio of the mean input and estimated spectra $<0.8$ or $>1.2$ in case of $g$ since measurement baselines of more than $20 \%$ are unlikely.

From the remaining points, those with a cost within $5 \%$ from the lowest cost were retained. If these points formed one group (maximal 0.01 difference in scaled parameters), the point with the lowest cost was selected as final PSD estimate. Else, no final estimate was given, because the solution was considered non-unique.

In case of $\mu_{s}$ and $\mu_{s}^{\prime}$, VF was estimated in a second optimization step based on nonnormalized spectra, since the particle concentration acts as a linear scaling factor in the independent scattering regime. PSD parameters were fixed at the values found in step one, leaving only VF undetermined in Eq. (2) instead of distribution parameters par 1 and par 2 . Five equidistant starting points were defined between the VF lower and upper boundary (Table 2).

As shown in Table 2, a separate set of parameter boundaries was defined for the three considered distribution types. To this end, the probability density functions were fitted directly to the reference PSDs. The lower boundaries were set at $70 \%$ of the minimum parameter values of the fitted distributions, the upper boundaries at $130 \%$ of the maximal values. In the optimization routine, all parameters were rescaled such that their range agrees with the interval 0.5-1.5
Table 2: Unscaled parameter boundaries.

\begin{tabular}{|cc|c|c|}
\hline & & lower & upper \\
\hline \multirow{2}{*}{ lognormal } & $\mu$ & -5.205 & 2.3629 \\
& $\sigma$ & 0.0151 & 1.292 \\
\hline \multirow{2}{*}{ normal } & $\mu$ & 0.00987 & 7.995 \\
& $\sigma$ & 0.00651 & 1.0632 \\
\hline \multirow{2}{*}{ Weibull } & $a$ & 0.0148 & 8.165 \\
& $b$ & 1.184 & 63.337 \\
\hline VF & & 0.00525 & 0.01625 \\
\hline
\end{tabular}

to reduce the effect of a different magnitude of the parameter values on the optimization.

\section{Goodness of fit of estimated PSDs}

The mode and full width at half maximum (FWHM) of all PSD estimates were calculated. For reference PSDs provided by the manufacturer, these parameters had to be derived approximately on interpolated PSDs since only tabular data of number based PSDs were provided (piecewise cubic interpolation to a particle diameter resolution of $0.2 \mathrm{~nm}$ ). Modes were identified at the maximum function value, while the FWHMs were calculated as the difference in particle size between the two points on the interpolated PSD with a function value equal to half the function value of the mode.

\section{Results and discussion}

\section{Simulated \& estimated BOP}

Figure 2 shows the average estimated $\mu_{s}$ spectra for the samples with a $1 \% \mathrm{v} / \mathrm{v}$ particle concentration, as well as the spectra simulated starting from the manufacturer's PSD. An overview of the estimated $\mu_{s}, g$ and $\mu_{s}^{\prime}$ spectra for all particle concentrations is as Fig. S-2, S-3 \& S-4. For (sub)micron particles, $\mu_{s}$ decreases monotonically with increasing wavelength, similar to the spectra reported by Michels et al. for submicron sized fat emulsions. ${ }^{32}$ In general, the wider the PSD, the more flattened the $\mu_{s}$ and $g$ spectra, as is clear for particles in the $3-12 \mu \mathrm{m}$ range (Fig. S-1 \& Fig. S-5). The estimated spectra show the same oscillating shape as those sim- 

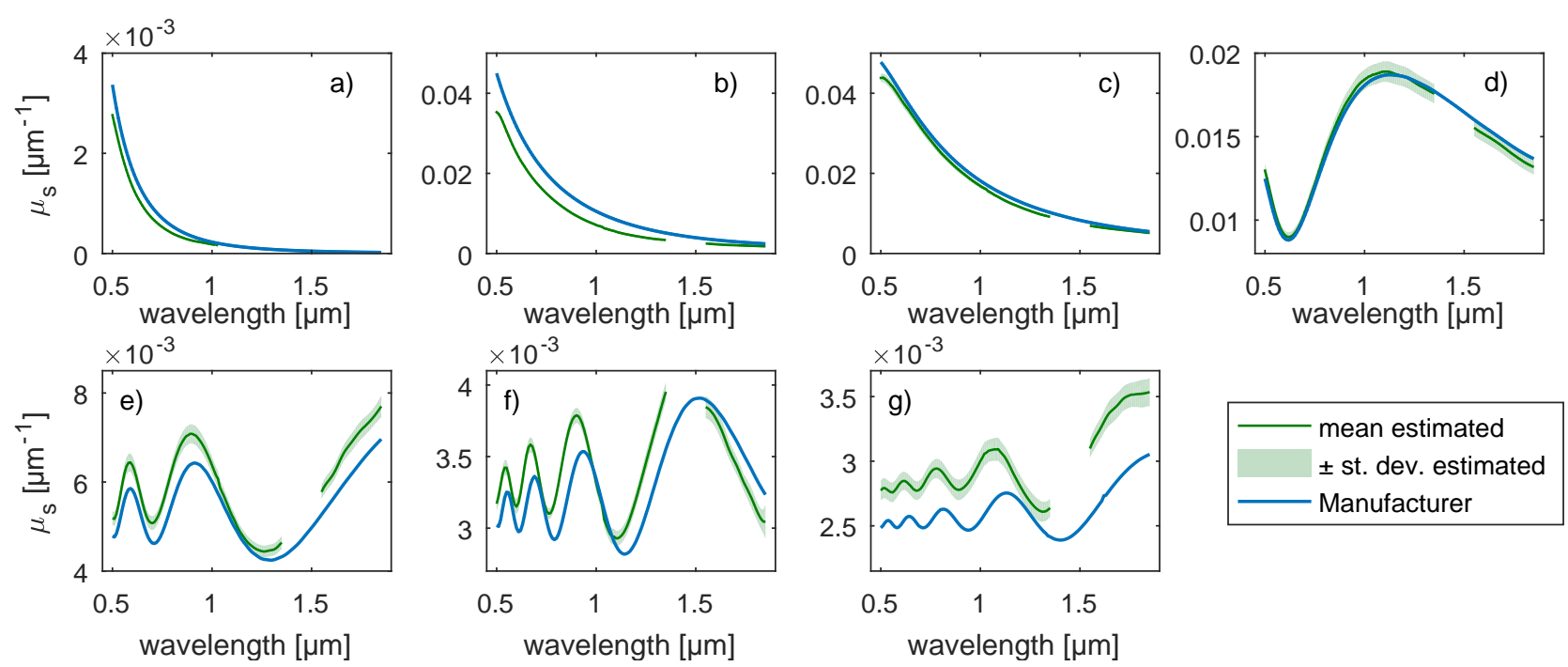

Figure 2: $\mu_{s}$ spectra estimated from double integrating sphere measurements obtained for samples with a $1 \% \mathrm{v} / \mathrm{v}$ particle concentration compared to the $\mu_{s}$ spectra simulated for the manufacturer PSD. a) $0.1 \mu \mathrm{m}, \mathrm{b}) 0.5 \mu \mathrm{m}$, c) $1 \mu \mathrm{m}$, d) $3 \mu \mathrm{m}$, e) $6 \mu \mathrm{m}$, f) $10 \mu \mathrm{m}, \mathrm{g}) 12 \mu \mathrm{m}$.

ulated for the manufacturer's PSD, which suggests that the PSD estimates are likely to be in line with the more narrow references. Compared to $\mu_{s}, \mu_{s}^{\prime}$ spectra are rather flat as the oscillations in $\mu_{s}$ and $g$ cancel out one another. ${ }^{32}$

The small discrepancy in position of the oscillations in the spectra of 6,10 and $12 \mu \mathrm{m}$ particles might be due to a minor error in the refractive index or the reference PSDs used in the forward simulations. However, since the discrepancy seems more like a translation of the spectra, rather than an incorrect spacing of the oscillations, a small error on the refractive index seems to be most likely. Earlier work indicated that a too large particle refractive index moves $\mu_{s}$ and $g$ spectra to larger wavelengths, with also an downward shift in $g .{ }^{18}$ Additional simulations with a baseline of $-0.02,-0.01$ and +0.02 on the real particle refractive index indicated that a slightly lower value would decrease the baseline mismatch between simulated and estimated $g$ spectra for the $10-12 \mu \mathrm{m}$ particles. However, for the $3 \mu \mathrm{m}$ and $6 \mu \mathrm{m}$ samples, the oscillations were positioned more correct when using the current values. Since all particles were believed to have the same refractive index and none of the baselines provided a consistent improvement for all particle sizes, no corrections on the refractive index were made.

For $10 \& 12 \mu \mathrm{m}$ particles, an offset was no- ticed between the estimated and simulated $\mu_{s}$ spectra. Since $\mu_{s}$ is linearly proportional to the particle concentration, this could be the consequence of an incorrect sample concentration, e.g. due to sedimentation during sample preparation or pipetting errors. Settling of the (large) particles during the optical measurements could be counteracted be using a flowthrough cell instead of a closed cuvette. Secondly, it could be related to the particle refractive index. Polystyrene was considered transparent and thereby non-absorbing. However, light absorption by particles does slightly influence the scattering spectra, as was found by additional forward simulations with an imaginary part of the particle refractive index set at 0.0005 (cf. Ma et al. ${ }^{27}$ ). Although including absorption decreased the baseline error for $g$, it increased the mismatch between simulated and estimated $\mu_{s}$ spectra. The large variation in (complex) refractive index values available in literature and the clear sensitivity of BOP to the refractive index point out the difficulty of finding the most correct values.

Thirdly, the BOP calculation by inverse adding-doubling might have been less accurate for the largest particles, since the number of scattering events was rather low due to the low concentrations. These were chosen to avoid dependent scattering, but resulted in a low total 
reflectance compared to the total transmittance in case of the 6,10 and $12 \mu \mathrm{m}$ particles. These low optical depths may have resulted in an overestimation of $g .{ }^{15,33}$ Consequently, the light loss through the exit port of the reflectance sphere might be larger than accounted for, which could explain the observed overestimation of $g$. The baseline in $\mu_{s}$ might also be related to the lower scattering level, since errors in $\mu_{s}$ increase as scattering decreases. The internal radiances become less uniform and are therefore less accurately approximated in integral equations. ${ }^{34}$ Nevertheless, all baseline errors affect only VF estimations on $\mu_{s}$ and $\mu_{s}^{\prime}$ since all spectra were normalized for PSD estimation.

\section{PSDs estimated on $\mu_{s}$ and $g$ spectra}

Figure $3 \& 4$ present the PSD estimates based on respectively $\mu_{s}$ and $g$, while Fig. 5 provides a general overview on the estimated volume fractions, relative modes and relative FWHM values. For all PSD estimates for 1-12 $\mu \mathrm{m}$ particles, the shape and level of the calculated spectra matches well with the $\mu_{s}$ values obtained by inverse adding doubling. Estimated $1 \mu \mathrm{m}$ PSDs have a peak position in line with the reference PSDs, but those for the lowest concentrations are more wide and slightly shifted (Fig. 3a, Fig. 5b-c). For particles $>1 \mu \mathrm{m}$, all estimates are very consistent (Fig. 3b-e), and relatively accurate in peak position and FWHM (Fig. 5bc, more details in Table S-3 \& S-4). Only the $3 \mu \mathrm{m}$ estimates are consistently wider than the manufacturer's reference PSD and have a width more in line with the LD-latex reference PSD, as indicated by the low RMSE in Table S-2. Even though the PSDs are not able to perfectly capture the long right tail of the 10-12 $\mu \mathrm{m}$ reference PSDs, the clear signature of their $\mu_{s}$ spectra results in good estimates.

VF estimates varied in general only slightly due to baseline noise on the $\mu_{s}$ spectra (Fig. 5a, $R^{2}=93.13 \%$, Table S-5).

When $g$ is used as input, estimated peak positions are in line with the reference PSDs for 1-12 $\mathrm{\mu m}$ particles (Fig. 4). The modes of these estimates have a RSME comparable to those estimated on $\mu_{s}\left(\mathrm{RMSE}_{\mu_{s}}=0.1045 \mu \mathrm{m}, \mathrm{RMSE}_{g}\right.$
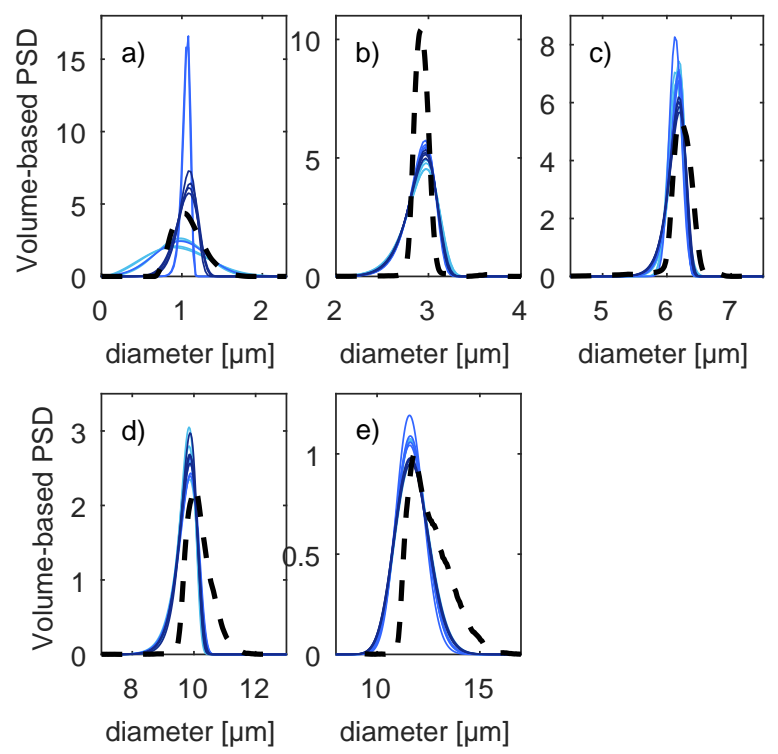

Figure 3: All PSD estimates based on $\mu_{s}$ for samples with a diameter around a) $1 \mu \mathrm{m} \mathrm{b}$ ) $3 \mu \mathrm{m}$, c) $6 \mu \mathrm{m}$, d) $10 \mu \mathrm{m}$ and e) $12 \mu \mathrm{m}$. Solid $=$ estimates, dashed $=$ manufacturer reference.

$=0.0978 \mu \mathrm{m})$, which is similar to the RMSECV reported by Chen et al. for mean particle diameter estimated on partial least squares models or based on multiplicative scatter correction coefficients (RMSECV range 0.0216-0.0721 $\mu \mathrm{m}) .^{8}$ The estimated PSD widths vary more amongst each other compared to estimations based on $\mu_{s}$ (Fig. 5e). This might be caused by the higher noise level on $g$ compared to $\mu_{s}$.

In the estimated $g$ spectra of $12 \mu \mathrm{m}$ particles, the oscillating pattern is almost completely overshadowed by noise due to the low reflectance signals in the double integrating sphere measurements. Despite the noisy $g$ spectra, a valid PSD estimate was selected for all but one samples of the $12 \mu \mathrm{m}$ particles, namely the third replicate of the $12 \mu \mathrm{m} 1 \%$ sample for which multiple local minima with a similar cost were found. Nevertheless, the PSD estimation routine would benefit from a more accurate $g$ determination for these particles. A better $g$ input spectrum would promote the selection of the correct solution when multiple local minima are present in the cost function, like for the example illustrated in Fig. 6a. The minimum at the expected particle size would then have a clearly lower cost than other minima, e.g. cor- 

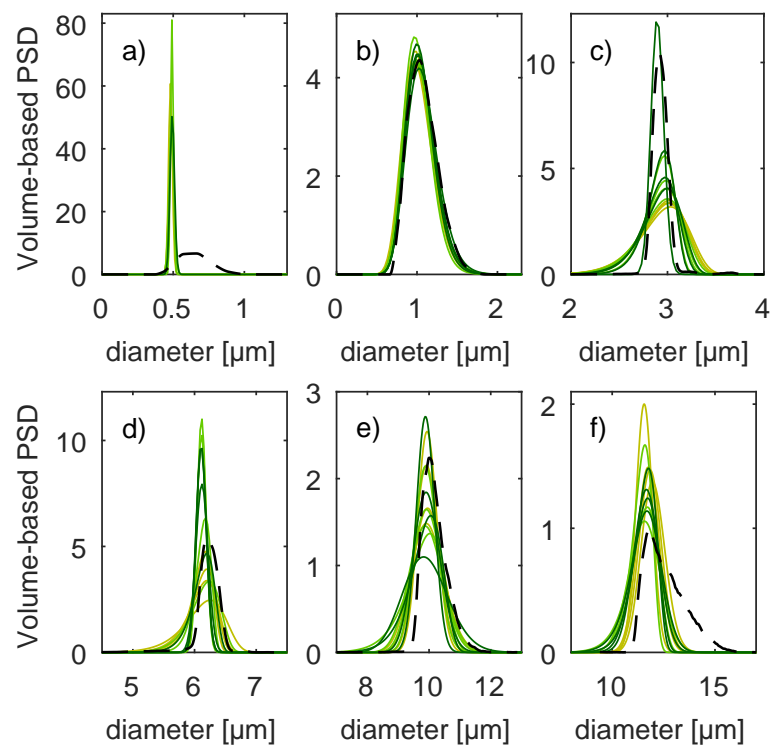

Figure 4: All PSD estimates based on $g$ for samples with a diameter around a) $0.5 \mu \mathrm{m}, \mathrm{b}$ ) $1 \mu \mathrm{m} \mathrm{c)} 3 \mu \mathrm{m}$, d) $6 \mu \mathrm{m}$, e) $10 \mu \mathrm{m}$ and f) $12 \mu \mathrm{m}$. Solid $=$ estimates, dashed $=$ manufacturer reference.

responding to a smooth spectrum matching the general trend of $g$. The latter would result in a too narrow PSD estimate shifted to micron size.

In contrast to the estimates in the previous paragraph, the PSD estimation was not successful for the $0.1 \mathrm{\mu m}$ samples. In case of $g$, this is likely caused by a low signal to noise ratio of the measurements, resulting in scattering spectra deviating strongly from the simulations (Fig. S-3). All estimates were discarded by the selection procedure because their cost was positive, which correspond to a sum of squared errors $>10$. The main scattering activity of the $0.1 \mathrm{\mu m}$ particles lies outside the considered wavelength range, in the UV region. This causes a drop in the discriminating power of the estimation routine, resulting in a widespread minimum in the cost function, as illustrated in Fig. 6b. In this case, a good fit of the spectral shape does no longer guarantee a correct PSD shape, since several combinations of distribution parameter values result in the same low cost.

The results for particles of $0.5 \mu \mathrm{m}$ are comparable to those of $0.1 \mu \mathrm{m}$, as again no valid estimate could be made based on $\mu_{s}$. When using a normal or Weibull distribution, the PSD was the most narrow possible and therefore discarded as invalid, while lognormal estimates on the other hand were the most wide distributions possible. In case of $g$, the spectra calculated for the PSD estimates do not fully match the noisy input spectra. Despite these disturbances having a direct impact on the estimated distribution parameters ${ }^{18}$, the PSD estimates for $0.5 \mu \mathrm{m}$ particles based on $g$ are better. However, they shifted to smaller sizes (mode $_{\text {est }}$ $\sim 0.49 \mu \mathrm{m}$ vs. mode $_{\text {ref }}=0.66 \mu \mathrm{m}$, Fig. $5 \mathrm{~d}$ ) to a peak position more in line with the LDnarrow and LD-latex reference (Table S1). Furthermore, they have an underestimated width compared to the manufacturer's PSD (Fig. 5e). In about $50 \%$ of the $0.5 \mu \mathrm{m}$ estimates on $g$ even the lower boundary for distribution width was reached. This shows the limitations of the PSD estimation routine for submicron particles in the considered wavelength range.

\section{PSDs estimated on $\mu_{s}^{\prime}$ spectra}

PSD estimates of $0.5 \mu \mathrm{m}$ particles on $\mu_{s}^{\prime}$ are stable, although more narrow and shifted to smaller sizes (Fig. 7, Fig. 8b-c). They resemble more the main LD-Narrow peak around $0.5 \mu \mathrm{m}$ (see RMSE on mode in Table S-1). The associated VF estimates are relatively correct for all samples (Fig. 8a), but less accurate than those based on $\mu_{s}\left(\mathrm{RMSE}_{\mu_{s}^{\prime}}=0.19 \% \mathrm{v} / \mathrm{v}\right.$ vs. $\left.\mathrm{RMSE}_{\mu_{s}}=0.05 \% \mathrm{v} / \mathrm{v}\right)$. For comparison, Chen et al. obtained a RMSECV of 0.058-0.09 wt.\% for particle concentrations estimated on a partial least squares model in a two-component system. $^{8}$

The smooth $\mu_{s}^{\prime}$ shape causes difficulties when estimating distribution widths, despite the good fits to the spectra. ${ }^{18}$ For $1-12 \mu \mathrm{m}$ particles, the majority of the PSD estimates (except for $6 \mu \mathrm{m}$ ) are shifted towards slightly smaller sizes and are clearly too narrow, accompanied by an underestimated VF (Fig. 8). For 10$12 \mu \mathrm{m}$ particles, the negative baseline on the estimated spectra compared to the simulations, aggravates the underestimation of the VF, and results for five samples $(10 \mu \mathrm{m} 0.75 \% 1-4,12 \mu \mathrm{m}$ 

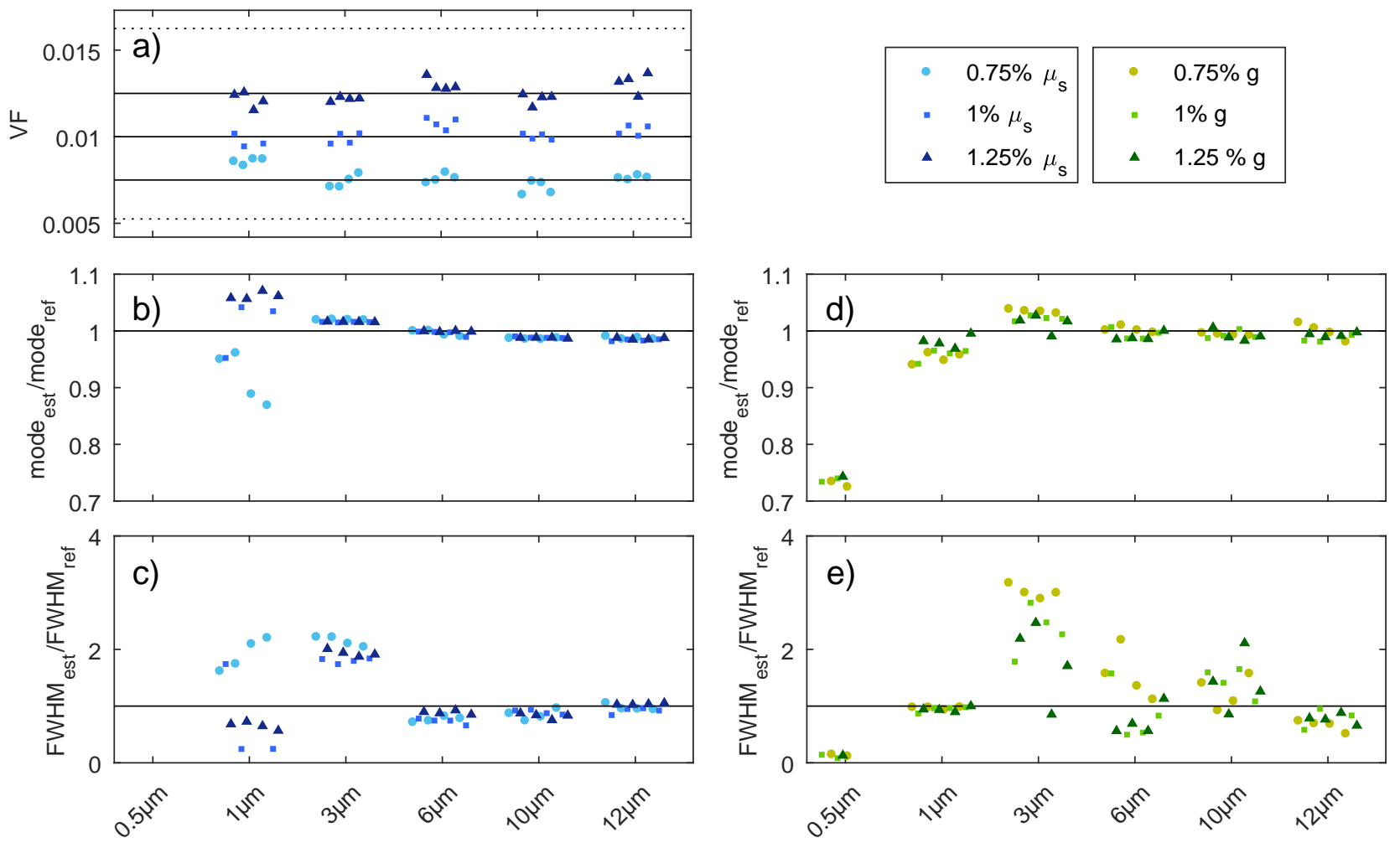

Figure 5: a)Volume fractions, b) relative modes and c) relative FWHM for PSDs estimated based on $\mu_{s}$. d) Relative modes and e) relative FWHM for PSDs estimated based on $g$. Full lines indicate the target values, dotted lines in a) indicate the upper and lower parameter boundaries.

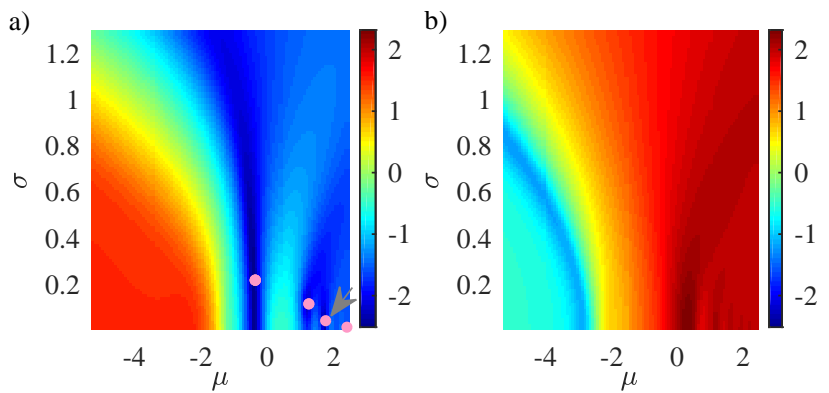

Figure 6: Illustrations of the cost as a function of the distribution parameters for lognormal PSD estimations for a) $12 \mu \mathrm{m} 1 \%$ on $g$ (replicate 3) and b) $0.1 \mu \mathrm{m} 0.75 \%$ on $\mu_{s}$ (replicate 1 ). In a), the pink dots indicate the retrieved solutions, the arrow marks the correct estimate.

$0.75 \% 4$ ) in an invalid PSD estimate due to reaching the lower VF boundary.

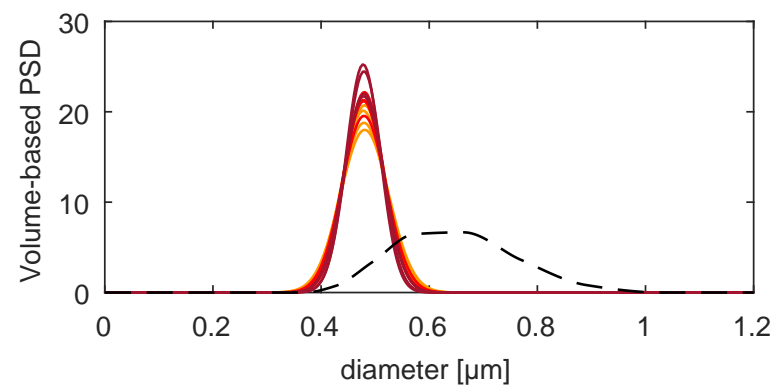

Figure 7: All PSD estimates for $0.5 \mu \mathrm{m}$ particles based on $\mu_{s}^{\prime}$. Solid $=$ estimates, dashed $=$ manufacturer reference.

\section{General discussion}

The accuracy of the BOP estimated from double integrating sphere measurements is crucial in order to obtain correct PSD estimates. These PSDs are selected to fit the scattering spectra as good as possible. So worst case, the 'best' estimate is the one that captures the noise on the spectra best, like the already mentioned example of a $12 \mu \mathrm{m}$ estimated on $g$. Preprocess- 

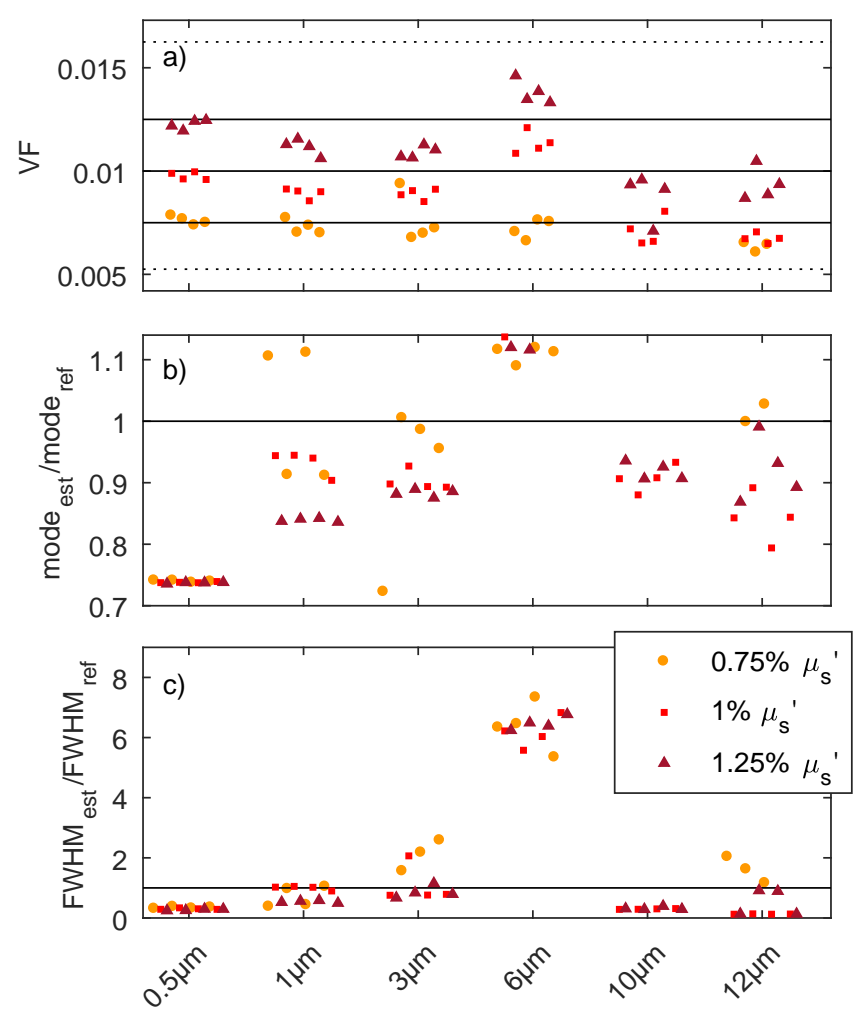

Figure 8: a) Volume fractions, b) relative modes and c) relative FWHM for PSDs estimated based on $\mu_{s}^{\prime}$. In c), sample $3 \mu \mathrm{m} 0.75 \% 1$ has a value of 47.4027 (not visible). Full lines indicate the target values, dotted lines in a) the parameter boundaries.

ing of the BOP spectra or averaging repeated measurements might help to reduce the effect of noise, as well as attributing a lower weight to or even discarding error prone wavelengths, as was done here for the region around $1450 \mathrm{~nm}$. Such regions could be determined based on theoretical knowledge (e.g. strong absorption peaks interfering with $\mu_{s}$, detection limits), or based on standard deviation values of repeated measurements or a thorough analysis of the set-up specific noise. Leaving out wavelengths may strongly influence the cost function shape: the number of local minima may change, a local minimum may become global.

Furthermore, the low particle concentrations and limited number of scattering events might not only contribute to the baseline mismatch between simulated BOP and those estimated from measurements in case of the large par- ticles, as mentioned before. It might also have cause particles located in the distribution tails to be underrepresented in the scattering events. Because of this, their contribution to the scattering spectrum might be lower than it should be and consequently, their fraction in the PSD can be underestimated. For submicron particles on the other hand, the very alike shape for $\mu_{s}$ and $\mu_{s}^{\prime}$ spectra of different particle sizes caused problems. This is similar to the very small difference in turbidity spectra of $30 \mathrm{~nm}$ polystyrene particles described by Glasse et al., causing an overestimated size for these small particles. ${ }^{35}$ Including wavelengths below $500 \mathrm{~nm}$ might enhance the quality of the PSD estimates, as the spectra show a more clear signature in that range.

In general, parameters associated with distribution width tend to have a smaller effect on the cost compared to parameters linked to mean particle size. This is also reflected in the large projected confidence regions on the estimated FWHM, compared to those on the mode (Table S-3 \& S-4). Such reduced sensitivity to distribution width was also observed by Saveyn et al. ${ }^{36}$ Moreover, the estimated distribution parameters are not completely independent. An interplay between the two parameters makes that small inaccuracies in the estimated peak position can be compensated by an altered distribution width. Overall, the estimated PSDs were relatively accurate, but the samples under investigation were close to being monodisperse. Therefore, additional validation on more polydisperse samples should make clear whether the estimation routine is indeed capable of providing correct distribution widths. Results obtained on simulated bulk scattering spectra using PSDs with ten different modes and each three distribution widths suggest no marked effect of distribution width should be expected. ${ }^{18}$ However, as PSDs become wider, their scattering spectra become more flat and therefore their particle size information might be lower. Such cases typically lack a characteristic spectral shape, and thus the PSD estimation might benefit from using simultaneously the information in the spectral shape and the absolute level, given that there are no baseline discrepancies 
between simulated and estimated spectra. This would lead to a one-step routine, in which the PSD parameters and particle concentration are estimated simultaneously.

The restrictive role of the parametric PSD shape should also be taken into account. For example, the 10-12 $\mathrm{mm}$ PSD have a right tail that cannot be represented correctly by the selected probability density functions. Regarding the more symmetrical PSDs, the majority of the $1 \mathrm{\mu m}$ samples were fitted by a lognormal distribution (63\%), while the middle-range particles of 3 and $6 \mu \mathrm{m}$ were most suited by a Weibull distribution (respectively $81 \%$ and $78 \%$ of the cases). For a closer comparison of the presented estimation routine with the state-ofthe-art particle sizing techniques, a shape dependent approach could also be applied on the raw data measured with LD and DLS. Nevertheless, developing and testing new data processing techniques for LD and DLS was not the scope of this study. To provide more flexibility in the PSD shape, it might be opportune to try a three parameter distribution, use a weighted combination of two probability density functions, or even a shape independent PSD estimation. ${ }^{35,37,38}$ Such increased flexibility in PSD shape is also necessary when estimating bimodal PSDs. A first step in that direction would be to adapt the current PSD estimation routine by allowing it to make a weighted combination of two probability density functions. A set of bimodal samples for validation could be created by mixing two particle sizes of monomodal polystyrene particles in varying proportions.

Notwithstanding the possible improvements on the quality of the measurements and the need for further validation on more polydisperse and even bimodal PSDs, the PSD estimation routine demonstrates the value of bulk scattering spectra for particle size retrieval. The insightful way of separating scattering and absorption allows to estimate a complete PSD, with additional VF in case of $\mu_{s}$ and $\mu_{s}^{\prime}$. In contrast to DLS or LD measurements, it allows to measure the samples without severe dilution.

\section{Conclusion}

The feasibility of estimating particle size distributions from experimentally determined bulk scattering spectra was demonstrated for monomodal polystyrene suspensions. PSD estimates were compared to reference measurements based on dynamic laser light scattering, laser diffraction and electrical sensing zone.

For particles of 1-12 $\mu \mathrm{m}$, good estimates were obtained based on $\mu_{s}$ and $g$. The estimated distribution width varied slightly more when estimates were made on $g$ spectra compared to $\mu_{s}$. The characteristic oscillating shape of these spectra for 6-12 $\mu \mathrm{m}$ allowed relatively accurate PSD estimates, even though the $g$ spectra of the $12 \mu \mathrm{m}$ samples were very noisy. Nevertheless, the parametric monomodal distribution was not able to capture the full asymmetry of the reference PSDs. VF estimates on $\mu_{s}$ were overall relatively accurate $\left(R^{2}=93.13 \%\right)$.

For submicron particles, the considered wavelength range provided insufficient discriminating power. This caused a broad region of low cost instead of a well-defined optimum. Limited information on distribution width was also a problem for all PSD estimations based on $\mu_{s}^{\prime}$. Even if the $\mu_{s}^{\prime}$ spectra were fitted correctly in the optimization, PSD estimates had a shifted mode and were mainly too narrow. Overall, the presented results show the potential of extracting particles size and concentration from pure scattering spectra, with most promising results if $\mu_{s}$ or $g$ are used.

Acknowledgement This research was funded by the Institute for the Promotion of Innovation though Science and Technology in Flanders (IWT, grant 141687) and Research Foundation-Flanders (FWO, grant 12K3916N). The authors wish to thank Jeroen Jordens (KU Leuven), Hilde Jans (Imec) and Daniël Vermeulen (KU Leuven) for helping with the particle size measurements. 
Supporting Information Available

The following files are available free of charge.

Figure S-1: Reference PSDs.

Figure S-2: Mean $\mu_{s}$ from measurements.

Figure S-3: Mean $g$ from measurements.

Figure S-4: Mean $\mu_{s}^{\prime}$ from measurements.

Figure S-5: Simulated $\mu_{s}$ spectra (all ref. PSDs).

Table S-1: RSMSE of modes (all ref. PSDs).

Table S-2: RSMSE of FWHM (all ref. PSDs).

Table S-3: Estimated modes with projected 95\% confidence regions.

Table S-4: Estimated FWHM with projected 95\% confidence regions.

Table S-5: Estimated VF with $95 \%$ confidence interval.

\section{References}

(1) Xu, R. Particuology 2015, 18, 11-21.

(2) Bowen, P. J. Dispersion Sci. Technol. 2002, 23, 631-662.

(3) Malloy, A.; Carr, B. Part. Part. Syst. Charact. 2006, 23, 197-204.

(4) Merkus, H. G. Particle Size Measurements; Springer, 2009; p 533.

(5) Huang, H.; Yu, H.; Xu, H.; Ying, Y. J. Food Eng. 2008, 87, 303-313.

(6) Aernouts, B.; Van Beers, R.; Watté, R.; Huybrechts, T.; Lammertyn, J.; Saeys, W. J. Dairy Sci. 2015, 98, 6727-38.

(7) Sarraguça, M. C.; Cruz, A. V.; Amaral, H. R.; Costa, P. C.; Lopes, J. A. Anal. Bioanal. Chem. 2011, 399, 2137-2147.

(8) Chen, Y. C.; Thennadil, S. N. Anal. Chim. Acta 2012, 746, 37-46.

(9) Higgins, J. P.; Arrivo, S. M.; Thurau, G.; Green, R. L.; Bowen, W.; Lange, A.; Templeton, A. C.; Thomas, D. L.; Reed, R. A. Anal. Chem. 2003, 75, 1777-1785.
(10) Flores-Cerrillo, J.; MacGregor, J. F. Ind. Eng. Chem. Res. 2002, 41, 1805-1814.

(11) Chen, Y. C.; Foo, D.; Dehanov, N.; Thennadil, S. N. Anal. Bioanal. Chem. 2017, 409, 6975-6988.

(12) Houben, C.; Nurumbetov, G.; Haddleton, D.; Lapkin, A. A. Ind. Eng. Chem. Res. 2015, 54, 12867-12876.

(13) Porfire, A.; Rus, L.; Vonica, A. L.; Tomuta, I. J. Pharm. Biomed. Anal. 2012, 84, 285-292.

(14) Steponavičius, R.; Thennadil, S. N. Anal. Chem. 2009, 81, 7713-7723.

(15) Pickering, J. W.; Prahl, S. A.; van Wieringen, N.; Beek, J. F.; Sterenborg, H. J. C. M.; van Gemert, M. J. C. Appl. Opt. 1993, 32, 399-410.

(16) Lu, R. Light Scattering Technology for Food Property, Quality and Safety Assessment ; CRC Press (Florida, United States), 2016; p 483.

(17) Steponavičius, R.; Thennadil, S. N. Anal. Chem. 2011, 83, 1931-1937.

(18) Postelmans, A.; Aernouts, B.; Saeys, W. Opt. Express 2018, 26, 15015-15038.

(19) Mastersizer 3000 user manual. 2011.

(20) Malvern Panalytical, Zetasizer Nano ZS. 2018; https: //www.malvernpanalytical.com/ en/products/product-range/ zetasizer-range/ zetasizer-nano-range/ zetasizer-nano-zs.

(21) Beckman Coulter, Multisizer 3 Coulter Counter. 2018; https://www. beckman.com/en/ cell-counters-and-analyzers/ multisizer-3.

(22) Malvern Panalytical, Mastersizer 3000. 2018; https:// www . malvernpanalytical.com/ 
en/products/product-range/

mastersizer-range/

mastersizer-3000.

(23) Aernouts, B.; Watté, R.; Van Beers, R.; Delport, F.; Merchiers, M.; De Block, J.; Lammertyn, J.; Saeys, W. Opt. express 2014, 22, 20223-20238.

(24) Segelstein, D. J. The complex refractive index of water. Ph.D. thesis, 1981.

(25) Sultanova, N.; Kasarova, S.; Nikolov, I. Acta Phys. Pol. A 2009, 116, 585-587.

(26) Polyanskiy, M. Optical constants of Polystyrene (PS). 2016; https: //refractiveindex.info/?shelf= organic $\{\backslash \&\}$ book=polystyren $\{\backslash \&$ \}page $=$ Sultanova.

(27) Ma, X.; Lu, J. Q.; Brock, R. S.; Jacobs, K. M.; Yang, P.; Hu, X.-H. Phys. Med. Biol. 2003, 48, 4165-4172.

(28) Inagaki, T.; Arakawa, E. T.; Hamm, R. N.; Williams, M. W. Phys. Rev. B 1977, 15, 3243-3253.

(29) van de Hulst, H. C. Light scattering by small particles; Structure of matter series; Wiley: New York (N.Y.), 1957; p 470.

(30) Aernouts, B.; Zamora-Rojas, E.; Van Beers, R.; Watté, R.; Wang, L.; Tsuta, M.; Lammertyn, J.; Saeys, W. Opt. Express 2013, 21, 32450-32467.

(31) Prahl, S. a. Everything I think you should know about inverse adding doubling. 2011; http: //omlc.ogi.edu/software/iad.

(32) Michels, R.; Foschum, F.; Kienle, A. Opt. Express 2008, 16, 5907-5925.

(33) Pickering, J. W.; Moes, C. J. M.; Sterenborg, H. J. C. M.; Prahl, S. A.; van Gemert, M. J. C. J. Opt. Soc. Am. 1992, 9, 621-631.

(34) Prahl, S. A.; van Gemert, M. J. C.; Welch, A. J. Appl. Opt. 1993, 32, 559568 .
(35) Glasse, B.; Riefler, N.; Fritsching, U. J. Spectrosc. 2015, 2015, 1-10.

(36) Saveyn, H.; De Baets, B.; Thas, O.; Hole, P.; Smith, J.; Van Der Meeren, P. J. Colloid Interface Sci. 2010, 352, 593600.

(37) Eliçabe, G. E.; García-Rubio, L. H. J. Colloid Interface Sci. 1989, 129, 192-200.

(38) Sun, X.; Tang, H.; Dai, J. Opt. Express 2007, 15, 11507-11516. 


\section{Graphical TOC Entry}

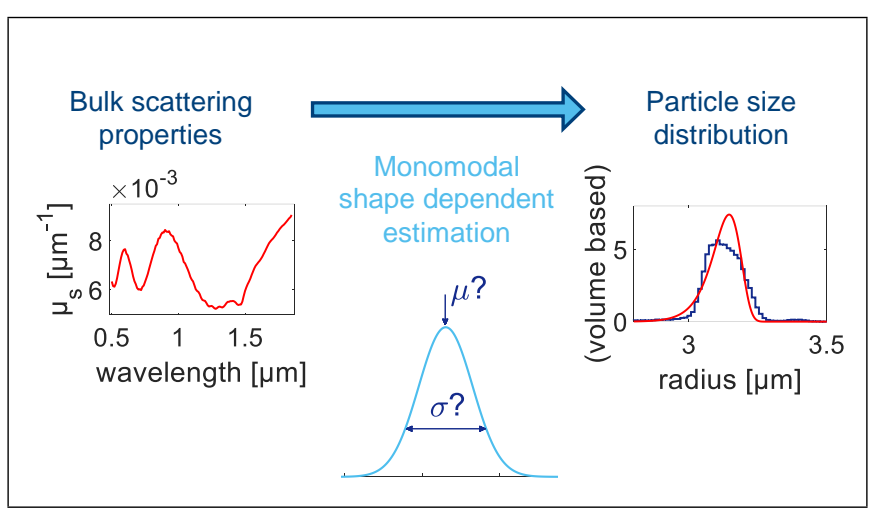

